DOI

\title{
ОТДАЛЕННЫЕ РЕЗУЛЬТАТЫ ОПЕРАТИВНОГО ЛЕЧЕНИЯ УЗЛОВЫХ ОБРАЗОВАНИЙ ЩИТОВИДНОЙ ЖЕЛЕЗЫ
}

\author{
๑Ш. А. Юсупов, З. Б. Курбаниязов, С. С. Давлатов, К. Э. Рахманов, Ф. А. Даминов \\ Самаркандский государственный медицинский институт, Республика Узбекистан
}

РЕзЮМЕ. Цель исследования - улучшение результатов хирургического лечения больных узловым зобом.

Материал и методы. В исследование был включен 281 пациент с заболеваниями щитовидной железы, госпитализированный в отделение хирургии клиники СамМИ для оперативного лечения с 2009 по 2016 год.

Результаты. Рецидив узлового зоба наблюдался у 9 больных $(4,7$ \%) в течение от 2 до 6 лет. Исходы операции были благополучными, и у 30 \% этих больных были выявлены атипичные клетки в противоположной доле щитовидной железы, несмотря на то, что клинически она выглядела интактной.

Выводы. На основании изучения отдаленных результатов лечения больных узловым зобом определен оптимальный объем хирургического вмешательства при различных морфологических формах узлового зоба. Адекватными объемами оперативного лечения являются гемиструмэктомия, предельно субтотальная резекция щитовидной железы и тиреоидэктомия. При проведении адекватной заместительной тиреоидной терапии резко снижается число рецидивов после проведенных оперативных вмешательств. Наибольший процент $(4,7 \%)$ рецидивов, независимо от морфологической формы узлового зоба, выявлен при проведении экономной резекции и энуклеации узла щитовидной железы.

КЛЮЧЕВЫЕ СЛОВА: узловой зоб; струмэктомия; отдаленные результаты; рецидив.

Вступление. В настоящее время в литературе имеются противоречивые данные о факторах, влияющих на прогноз лечения больных узловым зобом. Так, одни авторы утверждают, что определяющими в прогнозе возникновения рецидива узлового зоба являются гистологические изменения в щитовидной железе [3, 4], другие считают основными факторами объем оперативного вмешательства и адекватность проводимой тиреоидной терапии $[1,2,5]$. Поэтому большой интерес представляет определение влияния различных факторов на частоту возникновения рецидива узлового зоба на основании анализа отдаленных результатов хирургического лечения больных узловым зобом и разработка алгоритма индивидуального прогноза для выявления возможного рецидива в отдаленном периоде. Именно отдаленные результаты являются объективным критерием правильного выбора тактики лечения больных узловым зобом.

Цель исследования - улучшение хирургического лечения больных узловым зобом.

Материал и методы исследования. В исследование был включен 281 пациент с заболеваниями щитовидной железы, госпитализированный в отделение хирургии клиники СамМИ для оперативного лечения с 2009 по 2016 год. Среди пациентов были 253 (90,03 \%) женщины и 28 (9,97\%) мужчин. Средний возраст обследованных соста-

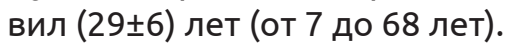

Больных вызывали активным способом - с помощью писем, также был изучен архивный материал клиники СамМИ. У всех больных тщательно изучали анамнез заболевания. Особое внима- ние было уделено длительности болезни, медикаментозному лечениюпрепаратами тиреоидных гормонов после операции. Были изучены такие показатели как объем операции, осложнения, исходы, ближайшие и отдаленные результаты хирургического метода лечения заболеваний щитовидной железы.

Всем больным проводили общеклиническое обследование, включающее пальпацию щитовидной железы, аускультацию сердца и сосудов, ультразвуковое исследование (УЗИ) щитовидной железы и зон регионарного лимфооттока в режиме реального времени (исходно и в различные сроки после операции), определение уровня тиреотропного гормона (ТТГ) (исходно и в различные сроки после операции), гистологическое исследование удаленного препарата проводили всем больным.

Степень увеличения щитовидной железы оценивали по классификации Николаева О. В. на основании УЗИ и пальпации щитовидной железы. у 71 (25,3 \%) больного диагностирован узловой зоб II-III степени, у 210 (74,7 \%) - узловой зоб IV-V степени. По патоморфологической форме (по Пенчеву) диффузный зоб выявлен у 44 (15,69\%) больных, узловой зоб -у 192 (68,3\%), смешанный 306 - у 45 (16,01\%). Распределение больных по степени увеличения и патоморфологической форме заболеваний щитовидной железы представлено в таблице 1.

На основании гормональных исследований крови (определение содержания трийодтиронина, тироксина, тиреотропного гормона), а также клинической картины у пациентов был установлен тиреоидный статус (табл. 2). 
Огляди літератури, оригінальні дослідження, погляд на проблему

Таблица 1. Характер заболеваний и степень увеличения щитовидной железы по классификации Николаева О.В.

\begin{tabular}{|c|c|c|c|c|c|c|c|}
\hline \multirow{2}{*}{\multicolumn{2}{|c|}{ Характер заболевания }} & \multicolumn{6}{|c|}{ Степень увеличения щитовидной железы } \\
\hline & & ІІ степень & III степень & IV степень & V степень & & его \\
\hline \multicolumn{2}{|c|}{ Диффузный токсический зоб } & - & 13 & 25 & 6 & \multicolumn{2}{|c|}{44} \\
\hline \multirow{4}{*}{ Узловой зоб } & токсическая аденома & - & 4 & 3 & - & 7 & \multirow{4}{*}{192} \\
\hline & нетоксический зоб & 1 & 27 & 69 & 1 & 98 & \\
\hline & кистозный зоб & - & 8 & 74 & 3 & 85 & \\
\hline & с-г щитовидной железы & - & 1 & 1 & - & 2 & \\
\hline \multicolumn{2}{|c|}{ Смешанный зоб } & - & 17 & 27 & 1 & \multicolumn{2}{|c|}{45} \\
\hline \multicolumn{2}{|l|}{ Всего } & 1 & 70 & 199 & 11 & \multicolumn{2}{|c|}{281} \\
\hline
\end{tabular}

Таблица 2. Функциональное состояние щитовидной железы у обследуемых больных

\begin{tabular}{|l|c|c|}
\hline \multicolumn{1}{|c|}{ Тиреоидный статус } & Количество & $\%$ \\
\hline Гипертиреоз & 51 & 18,2 \\
\hline Эутиреоз & 177 & 62,9 \\
\hline Гипотиреоз & 53 & 18,9 \\
\hline Всего & 281 & 100 \\
\hline
\end{tabular}

Оперативному лечению подверглись 278 больных, 3 больных не оперированы из-за тяжелого соматического состояния. Сроки оперативного вмешательства зависели от функционального состояния щитовидной железы. 48 (17,1\%) пациентов подверглись оперативному лечению после снятия тиреотоксикоза до эутиреоза (34) и гипотиреоза (14) в течение от 2 до 9 месяцев. 230 (81,8 \%) больным с эу- и гипотиреоидным статусом оперативные вмешательства были выполнены сразу.

Объем оперативного вмешательства зависел от патоморфологической формы зоба. Тотальная тиреоидэктомия была выполнена 7 (2,5 \%) пациентам при диффузном токсическом зобе и злокачественной опухоли левой доли щитовидной железы, соответственно у 5 и 2 больных. Субтотальная резекция щитовидной железы была выполнена 81 (28,8\%) больному с диффузным и смешанным зобом. у большинства боль- ных 190 (67,6\%) (табл. 3) объем оперативного вмешательства ограничился одной долей щитовидной железы.

Результаты и обсуждение. Частота возникновения осложнений во время операции на щитовидной железе или в раннем послеоперационном периоде напрямую связана с опытом хирурга, количеством операций, выполняемых им в год. В таблице 4 представлены данные по частоте ранних осложнений хирургического метода лечения заболеваний щитовидной железы нашей клиники.

В отдаленном послеоперационном периоде удалось проследить 193 из 278 пациентов, что составило 69,4 \%. Сроки наблюдения больных после операции составили от 1 года до 5 лет, результаты представлены в таблице 5 .

После вызова больных мы провели полный объем обследования, включающий клинический осмотр, пальпацию, оценку тиреоидного статуса

Таблица 3. Объем оперативного вмешательства в зависимости от патоморфологической формы зоба

\begin{tabular}{|l|c|c|c|c|c|}
\hline \multirow{2}{*}{$\begin{array}{c}\text { Объем оперативного } \\
\text { вмешательства }\end{array}$} & \multicolumn{5}{|c|}{ Патоморфологическая форма } \\
\cline { 2 - 6 } & диффузный зоб & узловой зоб & смешанный зоб & всего & \% \\
\hline Тотальная тиреоидэктомия & 5 & 2 & - & 7 & 2,5 \\
\hline $\begin{array}{l}\text { Субтотальная резекция } \\
\text { щитовидной железы }\end{array}$ & 36 & - & 45 & 28,8 \\
\hline Гемитиреоидэктомия & - & 156 & - & 156 & 55,5 \\
\hline $\begin{array}{l}\text { Субтотальная резекция одной } \\
\text { доли щитовидной железы }\end{array}$ & - & 11 & - & 3,9 \\
\hline $\begin{array}{l}\text { Энуклеация кисты из } \\
\text { щитовидной железы }\end{array}$ & - & 23 & - & 23 & 8,2 \\
\hline Не оперированные & 3 & - & - & 3 & 1,1 \\
\hline Всего & 44 & 192 & 45 & 281 & 100 \\
\hline
\end{tabular}


Огляди літератури, оригінальні дослідження, погляд на проблему

Таблица 4. Частота ранних осложнений хирургического метода лечения заболеваний щитовидной железы.

\begin{tabular}{|c|c|c|c|c|c|c|c|c|c|}
\hline \multirow{3}{*}{\multicolumn{2}{|c|}{ Диагноз }} & \multicolumn{7}{|c|}{ Вид осложнений } & \multirow[b]{3}{*}{ Всего } \\
\hline & & \multirow[b]{2}{*}{ 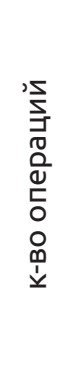 } & \multirow[b]{2}{*}{ 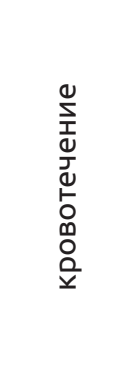 } & \multirow{2}{*}{ 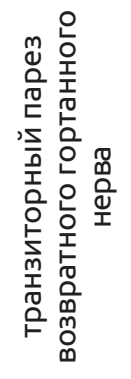 } & \multirow{2}{*}{ 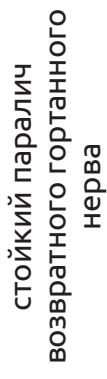 } & \multirow{2}{*}{ 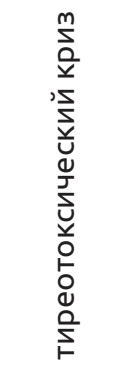 } & \multicolumn{2}{|c|}{ гипопаратиреоз } & \\
\hline & & & & & & & 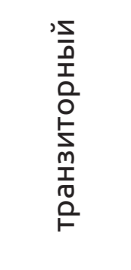 & 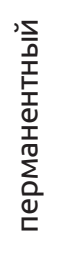 & \\
\hline \multicolumn{2}{|c|}{$\begin{array}{l}\text { Диффузный } \\
\text { токсический зоб }\end{array}$} & 41 & $7-17,1 \%$ & $2-4,9 \%$ & - & $1-2,4 \%$ & $2-4,9 \%$ & - & $12-29,3 \%$ \\
\hline \multirow{4}{*}{ 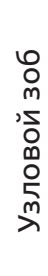 } & $\begin{array}{l}\text { токсическая } \\
\text { аденома }\end{array}$ & 7 & - & - & - & - & - & - & - \\
\hline & $\begin{array}{l}\text { нетоксический } \\
306\end{array}$ & 98 & $3-3,1 \%$ & - & - & - & - & - & $3-3,1 \%$ \\
\hline & кистозный зоб & 85 & $2-2,3 \%$ & - & - & - & - & - & $2-2,35 \%$ \\
\hline & $\begin{array}{l}\text { с-г щитовидной } \\
\text { железы }\end{array}$ & 2 & - & $1-50 \%$ & - & - & - & - & $1-50 \%$ \\
\hline \multicolumn{2}{|c|}{ Смешанный зоб } & 45 & $1-2,2 \%$ & $2-4,44 \%$ & - & - & $1-2,22 \%$ & - & $4-8,9 \%$ \\
\hline \multicolumn{2}{|c|}{ Всего } & 278 & $13-4,7 \%$ & $5-1,8 \%$ & - & $1-0,36 \%$ & $3-1,15$ & - & $22-7,9 \%$ \\
\hline
\end{tabular}

Таблица 5. Сроки наблюдения больных после операции.

\begin{tabular}{|l|c|c|c|c|}
\hline Сроки наблюдения & до 1 года & 3 года & 5 лет & Всего \\
\hline Количество больных & 89 & 68 & 36 & 193 \\
\hline
\end{tabular}

с помощью определения ТТГ, тиреоидных гормонов крови и рефлексометрии. Согласно полученным результатам было выявлено, что в состоянии эутиреоза находились 174 больных (90,2%), легкая степень гипотиреоза обнаружена у 6 $(3,1 \%)$, средней степени тяжести гипотиреоз у 8 $(4,1 \%)$, гипотиреоз тяжелой степени обнаружен у 5 больных (2,6\%), Следует отметить, что тяжелая степень послеоперационного гипотиреоза наблюдалась в группе больных, которым производилась тотальная тиреоидэктомия по поводу злокачественной опухоли щитовидной железы (2) и диффузного токсического зоба (3) (табл. 6).

Суммируя результаты клинических и гормональных исследований мы осуществили оценку отдаленных результатов хирургического лечения узловых форм зоба. Если учесть, что развитие гипотиреоза после операции на щитовидной железе является логическим и нормальным исходом операции, который легко компенсируется назначением тиреоидных гормонов, то согласно этому можно утверждать, что полное выздоровление больных после хирургического метода лечения узлового зоба наблюдается у 182 (94,3 \%) больных (табл. 7).

Рецидив узлового зоба наблюдался у 9 больных (4,7 \%) в течение от 2 до 6 лет. Все больные с рецидивом узлового зоба не следовали рекомендациям эндокринолога аккуратно и не принимали тиреоидные гормоны после операции. Результаты тонкоигольной аспирационной биопсии совпали с окончательным гистологическим результатом во всех случаях, то есть у этих больных была подтверждена доброкачественность удаленного узла (узлов). Исходы операции были благополучными, и у 30 \% этих больных

Таблица 6. Состояние функции щитовидной железы в момент осмотра больных в различные сроки после операции на щитовидной железе (по данным клинических признаков).

\begin{tabular}{|c|c|c|c|c|}
\hline \multirow{2}{*}{$\begin{array}{c}\text { Состояние функции } \\
\text { щитовидной железы }\end{array}$} & \multirow{2}{*}{ Эутиреоз } & гипотиреоз & \multicolumn{3}{|c|}{ Гикой степени } & средней тяжести & тяжелой степени \\
\cline { 3 - 5 } & & $6(3,1)$ & $8(4,1)$ & $5(2,6)$ \\
\hline Количество больных (\%) & $174(90,2)$ & & \\
\hline
\end{tabular}


Огляди літератури, оригінальні дослідження, погляд на проблему

Таблица 7. Отдаленные результаты оперативного лечения заболеваний щитовидной железы

\begin{tabular}{|l|c|}
\hline \multicolumn{1}{|c|}{ Исходы } & Количество больных (\%) \\
\hline Выздоровление & $182(94,3)$ \\
\hline Рецидив узлового зоба & $9(4,67)$ \\
\hline Рак щитовидной железы (по данным окончательной гистологии) & $2(1,03)$ \\
\hline
\end{tabular}

были выявлены атипичные клетки в противоположной доле щитовидной железы, несмотря на то, что она клинически выглядела интактной.

Выводы. Частота послеоперационных осложнений хирургического лечения заболеваний щитовидной железы очень низкая: послеоперационное кровотечение - 4,7 \%, временный парез возвратного гортанного нерва - 5,8 \%, стойкий паралич возвратного гортанного нерва - 0 \%, транзиторный гипопаратиреоз - 1,15\%, перманентный гипопаратиреоз - 0 \%. На основании изучения отдаленных результатов лечения больныхузловымзобомопределеноптимальный объем хирургического вмешательства при различных морфологических формах узлового зоба. Адекватными объемами оперативного лечения являются гемиструмэктомия, предельно субтотальная резекция щитовидной железы и тиреоидэктомия. При проведении адекватной за- местительной тиреоидной терапии резко снижается число рецидивов после проведенных оперативных вмешательств. Наибольший процент рецидивов, независимо от морфологической формы узлового зоба, выявлен при проведении экономной резекции и энуклеации узла щитовидной железы (4,7%). Для профилактики послеоперационного рецидива узлового зоба и лечения послеоперационного гипотиреоза необходима заместительная терапия препаратами тиреоидных гормонов под контролем эндокринолога.

Перспективы дальнейших исследований. Полученные результаты могут служить для изучения отдаленных результатов лечения больных узловым зобом и тем самым помогут определить оптимальный объем хирургического вмешательства при различных морфологических формах узлового зоба.

\section{ЛИТЕРАТУРА}

1. Аристархов В. Г. Отдаленные результаты оперативного лечения доброкачественных заболеваний щитовидной железы у пожилых пациентов / В. Г. Аристархов, Н. В. Данилов // Российский медико-биологический вестник имени академика И. П. Павлова. - 2014. - № 1.

2. Балаболкин М. И. Фундаментальная и клиническая тиреоидология : руководство / М. И. Балаболкин, Е. М. Клебанова, В. М. Креминская. - М. : Медицина, 2007. -816 c.

3. Хирургическая тактика лечения диффузно- токсического зоба / Ф. А. Даминов [и др.] // Академический журнал Западной Сибири. - 2013. - Т. 9, №. 1. - C. 21.

4. Отдаленные результаты хирургического лечения доброкачественной очаговой патологии щитовиднойжелезы/Ю.М. Таращенко [и др.]//Международный эндокринологический журнал. - 2015. - № 3 (67).

5. Bolgov M. Y. Results of Long-Term Follow-Up of Patients with Benign Nodules of Thyroid Gland / M. Y. Bolgov, Y. N. Taraschenko, I. R. Yanchiy // International Journal of Endocrinology. - 2014. - № 2 (58). - P. 9-14.

\section{REFERENCES}

1. Aristarkhov, V.G., \& Danilov, N.V. (2014) Otdalennyye rezultaty operativnogo lecheniya dobrokachestvennykh zabolevaniy shchitovidnoy zhelezy u pozhilykh patsiyentov [Long-term results of operative treatment of benign thyroid diseases in elderly patients]. Rossiykiy mediko-biologicheskiy vestnik imeni akademika I.P. Pavlova - Russian Medical-Biological Journal named after Academician I.P. Pavlov, 1 [in Russian].

2. Balabolkin, M.I., Klebanova, E.M., Kreminskaya, V.M. (2007). Fundamentalnaya i klinicheskaya tireoidologiya (rukovodstvo) [Fundamental and clinical thyroidology (guidance)]. Moscow: Meditsyna [in Russian].

3. Daminov, F.A. (2013). Khirurgicheskaya taktika

lecheniya diffuzno-toksicheskogo zoba [Surgical tactics of treating diffuse-toxic goiter]. Academic Journal of Western Siberia, 9 (1), 21.

4. Taraschenko, Yu.M. (2015). Otdalennyye rezultaty khirurgicheskogo lecheniya dobrokachestvennoy ochagovoy patologii shchitovidnoy zhelezy [Remote results of surgical treatment of benign focal pathology of the thyroid gland]. Mezhdunarodnyi endokrinologicheskiy zhurnal - International Endocrinology Journal, 3 (67) [in Russian].

5. Bolgov, M.Y., Taraschenko, Y.N., \& Yanchiy, I.R. (2014). Results of long-term follow-up of patients with benign nodules of thyroid gland. International Journal of Endocrinology, 2 (58), 9-14. 


\title{
Огляди літератури, оригінальні дослідження, погляд на проблему \\ ВІДДАЛЕНІ РЕЗУЛЬТАТИ ОПЕРАТИВНОГО ЛІКУВАННЯ ВУЗЛОВИХ УТВОРІВ ЩИТОПОДІБНОї ЗАЛОЗИ
}

\author{
ОШ. А. Юсупов, З. Б. Курбаніязов, С. С. Давлатов, К. Е. Рахманов, Ф. А. Дамінов \\ Самаркандський державний медичний інститут, Самарканд, Республіка Узбекистан
}

РЕЗЮМЕ. Мета дослідження - покращення результатів хірургічного лікування хворих з вузловим зобом.

Матеріал і методи. У дослідження був включений 281 пацієнт із захворюваннями щитоподібної залози, госпіталізований у відділення хірургії Самаркандського державного медичного інституту для проведення оперативного лікування протягом 2009-2016 рр.

Результати. Рецидив вузлового зоба спостерігався у 9 випадках (4,7 \% хворих) у часовому проміжку від 2 до 6 років. Наслідки оперативного втручання були благополучними, у 30 \% пацієнтів були виявлені атипові клітини у протилежній частці щитоподібної залози, хоча клінічно вона виглядала інтактною.

Висновки. На основі вивчення віддалених результатів хірургічного лікування визначено оптимальний об'єм хірургічного втручання при різних морфологічних формах вузлового зоба. Адекватними об'ємами оперативного втручання визначено геміструмектомію, максимально субтотальну резекцію щитоподібної залози та тиреоїдектомію. При проведенні адекватної замісної терапії різко знижується число рецидивів після проведених оперативних втручань. Найбільший відсоток $(4,7$ \%) рецидивів, не залежно від морфологічної форми вузлового зоба, зафіксовано при проведенні економної резекції та енуклеації вузла щитоподібної залози.

КлючОВІ СлОВА: вузловий зоб; струмектомія; віддалені результати; рецидив.

\section{DISTANT RESULTS OF SURGICAL TREATMENT OF THYROID NODULES}

\section{OSh. A. Yusupov, Z. B. Kurbaniyazov, S. S. Davlatov, K. E. Rakhmanov, F. A. Daminov Samarkand State Medical Institute, Republic of Uzbekistan}

SUMMARY. The aim of the study - improving the results of surgical treatment of patients with nodular goiter.

Materials and Methods. The study included 281 patients with thyroid disease who were hospitalized in the Department of Surgery of the summit clinic for surgical treatment from 2009 to 2016.

Results. Recurrence of nodular goiter was observed in 9 patients (4.7\%) for 2 to 6 years. Outcomes of surgeries were successful, and in $30 \%$ of these patients, atypical cells were identified in the opposite lobe of the thyroid gland, despite the fact that it looked clinically intact.

Conclusions. The study of long-term results of treatment of patients with nodular goiter determined the optimal amount of surgery in different morphological forms of nodular goiter. Adequate volumes of surgical treatment are hemistrumectomy, extremely subtotal resection of the thyroid gland and thyroidectomy. In carrying out adequate thyroid replacement therapy dramatically reduced the number of relapses after the surgery. The highest percentage $(4.7 \%)$ recurrences regardless of the morphological form of nodular goiter was revealed during the economical resection and enucleation thyroid nodule.

Key words: nodular goiter; strumectomy; distant results; relapse. 